Voix et Images

volxetimages

\title{
Entretien avec Dany Laferrière
}

Jean Morency et Jimmy Thibeault

Volume 36, numéro 2 (107), hiver 2011

Dany Laferrière

URI : https://id.erudit.org/iderudit/1002439ar

Aller au sommaire du numéro

Éditeur(s)

Université du Québec à Montréal

ISSN

0318-9201 (imprimé)

1705-933X (numérique)

Découvrir la revue

Citer ce document

Morency, J. \& Thibeault, J. (2011). Entretien avec Dany Laferrière. Voix et

Images, 36(2), 15-23.

Ce document est protégé par la loi sur le droit d'auteur. L'utilisation des services d'Érudit (y compris la reproduction) est assujettie à sa politique d'utilisation que vous pouvez consulter en ligne.

https://apropos.erudit.org/fr/usagers/politique-dutilisation/
Cet article est diffusé et préservé par Érudit.

Érudit est un consortium interuniversitaire sans but lucratif composé de l'Université de Montréal, l'Université Laval et l'Université du Québec à Montréal. Il a pour mission la promotion et la valorisation de la recherche. https://www.erudit.org/fr/ 


\section{ENTRETIEN AVEC DANY LAFERRIÈRE}

$+++$

JEAN MORENCY

Université de Moncton

JIMMY THIBEAULT

Université de Moncton

VoIX ET IMAGES Lorsqu'on passe en revue l'ensemble de votre œuvre, surtout depuis L'odeur du café jusqu'à L'énigme du retour, on se rend compte que les textes s'enchaînent de manière naturelle. Aviez-vous déjà une idée précise de ce que serait votre œuvre au moment de publier Comment faire l'amour avec un Nègre sans se fatiguer? Ou est-ce que le projet de l'«Autobiographie américaine» vous est venu en cours d'écriture?

DANY LAFERRIĖRE C'est venu très étrangement, après Éroshima. En relisant mes notes, j'ai lu, un jour, comment Balzac avait été frappé par les liens qui unissaient ses romans et comment, en constatant ces liens, il en était venu à formuler le projet de sa Comédie humaine. D'où l'idée de mon «Autobiographie américaine». Je me suis dit: «Mais c'est vrai! Il y a, entre mes romans, des liens intéressants, mais je ne vais pas les forcer.» C'est-à-dire que si j'écris avec l'idée consciente que je suis en train de faire une grande tapisserie, je vais laisser maintenant les choses aller et regarder comment elles se tissent. Il y a beaucoup de thèmes qui créent ces liens entre les romans; l'immobilité, par exemple, qu'on retrouve dans L'odeur du café, avec la grand-mère qui est toujours assise, et dans Comment faire l'amour..., avec Bouba qui est toujours couché. Ou il y a les écrivains qui guident toujours la narration, comme Bashô, Whitman, Césaire ou Miron.

VoIX ET IMAGES Les figures d'écrivains sont en effet très présentes dans vos romans. DANY LAFERRIĖRE J'aime cheminer avec un écrivain, citer des livres, parce qu'ils me servent de repères. Quand je les vois quelque part, je suis rassuré. Le papier imprimé me rassure dans la vie, alors j'en mets dans mes livres. Il n'y a peut-être que dans L'odeur du café qu'il n'y en a pas. Dans Le goût des jeunes filles, on retrouve déjà le poète Magloire Saint-Aude, qui est le premier guide. Ces écrivains, je les appelle les "poissons-pilotes ». Et il y a tous les autres après. Il y a même Lao-Tseu dans Eroshima. L'écrivain devient ainsi un personnage de mon œuvre.

voIX ET IMAGES Un peu à la manière de Pays sans chapeau, vos romans semblent se situer entre le rêve et la réalité, entre un monde qui serait plutôt de l'ordre de l'imaginaire, de la fabulation, de la mémoire, et un monde davantage tourné vers le présent du narrateur. Cette opposition entre deux mondes s'est-elle imposée en cours d'écriture ou est-ce que vous saviez, dès le départ, qu'elle marquerait votre œuvre? 
DANY LAFERRIÉRE Non, je n'avais pas conscience de cela. Mais je pense que la grande fracture vient du fait que j'ai eu un choc quand j'ai quitté mon pays. C'est-à-dire que, brusquement, mon présent de l'indicatif est passé dans la mémoire. Quelque chose qui était hier au présent est devenu, du jour au lendemain, au passé définitif. Cela a contribué à créer dans mes romans un paysage violent, au sens d'une violence dans les différences, marqué par la chaleur et l'hiver. Le passage vers un pays sans chapeau, littéralement. Je pense que cela a eu un impact sur la structure de mon œuvre. En même temps, j'ai toujours été à la fois féminin et masculin: j'ai été élevé par des femmes, mais je n'ai jamais renié le monde des hommes. Il y a donc aussi ces deux mondes.

VoIX ET IMAGES L'énigme du retour serait un retour vers le pays rêvé, celui du passé? DANY LAFERRIĖRE Je dirais que la différence entre ce roman et mes autres livres, c'est qu'il est plus multiforme, plus sphérique. Il permet de retrouver un univers sphérique, au sens où ce n'est pas seulement le pays réel et le pays rêvé, c'est aussi celui de l'enfance, de la mémoire, autrement dit, un pays mystique. Il y a comme un tourbillon de choses qui, parce que le narrateur retourne vers le pays rêvé qui était en fait un pays réel quand il était en Haïti, ne sont plus rêvées. En même temps, elles appartiennent toujours à l'imaginaire, car, que l'on soit en Haïti ou pas, le monde mystique est un monde où, avec très peu de choses réelles, concrètes, on bascule dans un autre champ. Comme si le pays réel et le pays rêvé du narrateur devenaient un pays mystique, mais sans qu'il soit lui-même un mystique. C'est peut-être la raison pour laquelle les gens ont aimé lire le livre, parce qu'il n'y a, dans L'énigme du retour, aucun esprit militant. On a l'impression que le narrateur n'est tombé dans aucune potion magique, comme le nationalisme culturel. Dès que le lecteur constate, par exemple, que le narrateur souffre de diarrhée, il se dit: «Ha ! L'auteur n'est pas tombé dans le discours nationaliste.» D'ailleurs, le narrateur mentionne: «Le nationalisme peut abuser mon esprit, mais pas mes intestins. » Et cela est rassurant car, généralement, dans les livres écrits par des gens du Sud qui vivent au Nord, il y a toujours un moment où il y a un divorce avec le pays d'origine. Comme si l'auteur qui vit hors de son pays ne pouvait plus suivre et qu'il devait se contenter de regarder, admirativement, de loin. C'est aussi pour dire que tout individu, tout écrivain est étranger à son pays, parce qu'il ne peut pas observer ce pays s'il n'y est pas étranger. Il faut qu'il prenne une distance. Donc, c'est cela, la notion poétique qui rend l'affaire intéressante, il ne s'agit pas simplement de dire: «Je suis devenu étranger dans mon pays parce que je n'y suis pas allé depuis longtemps. » C'est une distance qui est prise jusqu'aux fibres les plus profondes.

VoIX ET IMAGES Le narrateur se pose peut-être comme étranger mais, en même temps, les autres personnages perçoivent en lui un dieu vaudou, donc faisant partie de l'espace mystique du pays.

DANY LAFERRIÈRE Le pays le considère pourtant comme un étranger, car un dieu est un étranger, mais un étranger qui nous habite tout en venant d'ailleurs, d'un Olympe. C'est intéressant de voir comment l'étranger peut être plus profondément ancré dans le pays que le natif. En même temps, c'est étrange parce que les gens qui sont dans le réel, dans la ville, le perçoivent comme un étranger, c'est-à-dire comme quelqu'un venant d'un lieu différent. Et les gens qui ne savent pas qu'il vient d'un autre pays le perçoivent comme un dieu, un loa, un dieu vaudou. C'est intéressant aussi pour 
analyser la notion de l'étranger qui, de plus en plus en Occident, est considéré comme un être inférieur. Il est non plus un barbare qui apporte le danger, mais un être inférieur qui a accepté les travaux les plus subalternes. Et là, dans L'énigme du retour, l'étranger a repris toute sa force comme un être qui plane, un être qui vient d'en haut. VoIX ET IMAGES Dans votre roman, il y a une coupure très nette entre la ville de Portau-Prince et la campagne haïtienne. Le narrateur est considéré comme étranger à Portau-Prince, mais il semble au diapason du paysage de la campagne. Cette coupure n'est cependant pas nouvelle, on la retrouve dans l'ensemble de votre œuvre. L'écriture semble se durcir lorsqu'il est question de Port-au-Prince, probablement en raison du contexte politique, alors que les romans qui se passent à la campagne font appel à un imaginaire davantage marqué par la douceur. On retrouve également cette coupure dans Pays sans chapeau, où le culte vaudou semble plus sympathique à la campagne qu'en ville.

DANY LAFERRIÈre Le vaudou à Port-au-Prince, c'était plutôt l'affaire des buveurs de sang, des gens qui en agressaient d'autres pendant la nuit. Il faut cependant reconnaître que mon rapport à la campagne est assez compliqué. Lorsque je parle de la campagne, ce n'est pas seulement de la campagne haïtienne, c'est aussi d'un mode de vie. C'est pour cela que si on analyse mes livres uniquement en termes de territoire, on se trompe. Il s'agit d'un mode de vie qui renvoie à l'enfance, qui a été pour moi heureuse, et qui obéit à des règles complètement différentes des problèmes de grandes villes comme Port-au-Prince ou Montréal, où l'on retrouve des problèmes de vitesse, de stress, d'agressivité, de faim, de maladie, de hiérarchie sociale. Ce sont des problèmes de survie. Alors que, dans ma tête, la campagne, la paysannerie, représente un autre mode de vie qui pose un lien plus fort avec le monde invisible et une harmonie qui n'est pas mystique. C'est un art de vivre que je qualifierais de plus sphérique. Quand on considère le monde de $\mathrm{Da}$, dans L'odeur du café ou Le charme des après-midi sans fin, on retrouve un monde clos, assez fermé, mais pas dans le sens de «fermeture», plutôt dans le sens d'«indépendant», «autonome». C'est une cellule complètement autonome qui n'a rien à voir avec le reste d'Haïti et qui est plus universelle. D'ailleurs, j'aimerais, un jour, écrire un petit livre où le narrateur serait un officier d'état civil, comme mon grand-père qui tenait les registres et qui était un féru de littérature grecque. Dans mon imagination, il a toujours vu Petit-Goâve comme un village grec où se retrouvaient Socrate et Platon, mais qui étaient devenus tout simplement des gens très ordinaires. Il leur donnait des noms comme ça parce que l'État haïtien avait demandé de changer les noms qui apparaissaient plus proches des noms indiens que des chrétiens. Alors, mon grand-père poussait les gens à prendre des noms comme Hippocrate ou Aristophane, et il a basculé, un peu comme Cervantès, dans ce monde-là, il a commencé à croire que ces paysans, souvent analphabètes, étaient en train de résoudre des problèmes de maïeutique, des problèmes philosophiques tout en continuant leur vie quotidienne. Donc, dans ma tête, ce monde de la haute civilisation grecque représente Petit-Goâve. Le centre de l'âge d'or de la Grèce, c'est ma grand-mère sur cette galerie avec une cafetière, qui offre du café aux gens qui passent et qui s'entretient avec eux. Pour moi, Petit-Goâve n'est pas du tout une vision idyllique ou nostalgique d'une petite ville de province, c'est un effort pour reconstruire le vieux rêve humain d'harmonie, d'intelligence, d'esprit et de philosophie, comme les Grecs l'ont tenté dans leur temps. 
voix ET IMAGES Vous avez parlé plus tôt de l'importance qu'a eue, pour votre écriture, l'obligation de quitter rapidement votre pays, de vous exiler. Pourtant, dans Chronique de la dérive douce, le personnage refuse l'argent supplémentaire donné aux exilés puisqu'il se définit non pas comme un exilé, mais comme un individu qui a fui son pays. Le narrateur du Cri des oiseaux fous, pour sa part, réalise, après plusieurs années au Nord, qu'il a pris l'accent de l'exil. On croirait donc que l'exil est plutôt de l'ordre de la temporalité que de la spatialité. À vos yeux, y a-t-il une différence entre la fuite et l'exil?

DANY LAFERRIĖRE Il y a deux ou trois choses dans ces vocables. Il ne faut pas oublier qu'il y a toujours deux narrateurs dans mes romans. Il y a un narrateur singulier, qui pourrait être moi, qui utilise des éléments autobiographiques liés à ma perception du monde. Et ce narrateur est en lutte contre un dictateur qui veut lui imposer un mode de vie. L'exil est une conséquence de la dictature. On ne s'exile pas, on vous met en exil. Mais ce narrateur a toujours refusé de se considérer en exil, préférant plutôt se définir comme un voyageur. Pendant longtemps, j'ai été attentif à cette différence entre le voyage et l'exil. Ensuite, il y a un narrateur collectif, qui porte le drame de ceux qui ont subi l'exil et qui, eux, ont fait le choix de se définir comme exilés. Je ne pouvais pas leur imposer ma notion du voyage, parce qu'en se disant exilés, ils revendiquent du même souffle la punition du dictateur, tout en faisant de lui un monstre. Si on est en voyage, il n'y a plus de dictateur. Mais pour ceux qui avaient une vision politique de l'affaire, c'était très important que le visage monstrueux du dictateur apparaisse sur la scène internationale. Je ne pouvais pas continuer à refuser le mot exil. Mais dans mon cas, il n'a jamais été question d'exil, parce que, dans ma lutte à moi, j'avais d'autres armes pour combattre le dictateur. Je pouvais ne pas le nommer. Mais tous ces gens, ébénistes, électriciens, maçons, chauffeurs de taxi ou médecins, ne pouvaient pas toujours compter sur ces armes que me confère l'écriture dans leur lutte politique et il leur fallait nommer le dictateur. Donc, par respect pour leur expérience, j'ai intégré la notion d'exil.

voix ET IMAGES Avec L'énigme du retour, avez-vous l'impression d'avoir écrit, même si le mot peut sembler assez fort, le grand roman américain? Cette tentation semble déjà présente dans Cette grenade dans la main du jeune Nègre est-elle une arme ou un fruit?, mais L'énigme du retour apparaît comme une œuvre qui vient compléter cette espèce de vision de l'expérience d'ensemble de la collectivité américaine, cette fois, à partir d'Haïti.

DANY LAFERRIÈRE Le grand roman américain est une invention américaine, un jeu, comme il y a le grand hamburger américain ou le plus gros melon du monde. Ceci étant dit, je pense que c'est la version américaine de ce qu'en Europe on appelle l'immortalité. Comme les Américains sont très concrets, ils ne peuvent pas dire un mot aussi vague qu'immortalité, alors ils appellent cela le grand roman américain, ce qui veut dire «atteindre l'immortalité». Toutefois, on ne peut pas écrire le grand roman américain sans l'aide du lecteur. C'est le lecteur qui va définir le grand roman américain. Mais il y a toujours eu cette idée chez moi, qui est liée non pas à l'immortalité, mais plutôt à la volonté de faire un pied de nez aux écrivains américains, états-uniens, qui voulaient écrire le grand roman américain en parlant, comme Philip Roth, de baseball. Je trouvais qu'il y avait là des zones assez restreintes et que je devais passer d'abord par des espaces-temps, comme l'enfance vécue dans un petit village d’Haïti, qui se trouve en 
Amérique. Il y a aussi Port-au-Prince situé dans un pays qui a souffert toutes les tribulations de l'Histoire depuis la grande douleur de l'esclavage. Il n'y a rien de plus américain qu'Haïti. Quand l'armée napoléonienne est débarquée à Saint-Domingue avec vingt-deux mille hommes et quatre-vingt-six navires de guerre pour rétablir l'esclavage, c'était pour une question d'argent. L'esclavage représentait alors la moitié du produit national brut français. On ne pouvait pas plaisanter avec cela quand on était en train de guerroyer avec l'Angleterre. Donc, il y a eu ce grand affrontement et cette grande injustice qui ont amené la grande douleur dans les ateliers de Saint-Domingue. De là, on arrive à Montréal, qui est la frontière de l'Europe et de l'Amérique. À l'intérieur même de cette ville, il y a la confrontation de langues, séparées par le boulevard SaintLaurent, de religions, catholique et protestante, de cultures et de colonisations faites par des semblables. On connaît la haine des semblables. Une guerre européenne entre l'Angleterre et la France vient se reproduire en Amérique du Nord. À Montréal, on est dans une ville des plus américaines qui soient. Dans Cette grenade..., on traverse le continent, on circule à travers les petites villes des États-Unis et aussi à New York. Et tout cela nous donne le grand roman américain. J'ai élargi le territoire en abordant des villes comme Port-au-Prince et Montréal. Ce sont des villes extrêmement précieuses pour la connaissance des problèmes de l'Amérique, parce que si le grand roman américain ne parle pas des problèmes de l'Amérique, s'il parle uniquement de la montée du capitalisme, comme beaucoup l'ont fait dans leur tentative, on passe à côté de quelque chose d'important. Dans ce sens-là, la boîte noire et la chaudière de l'« Autobiographie américaine», si on y regarde de près, peuvent apporter des éléments nouveaux.

VoIX ET IMAGES Pratiquement tous vos romans renvoient au présent, comme si le passé et l'avenir n'existaient que dans la mesure où ils sont énoncés au présent. Dans les romans qui se passent à Port-au-Prince, par exemple, le présent des personnages paraît prendre toute la place parce que ces derniers sont mis dans une situation de survie au quotidien. Et cela se reflète dans la narration, comme dans les romans de la mémoire, où il y a nettement un processus de remémoration. Comment percevez-vous cette temporalité? Est-ce que le passé, surtout, se présente comme une complétude, donc une construction, du présent par le sujet qui raconte?

DANY LAFERRIĖRE Tout est au présent, le passé comme l'avenir. C'est une décision que j'ai prise, de mettre tout au présent. En fait, le passé existe, l'avenir existe, mais ils sont mis au présent. Le passé est compris, en Amérique du Nord et en Europe, à travers la notion d'héritage. Sans cela, on est dans l'imaginaire total. Le passé existe parce que la maison où j'habite a appartenu à mon grand-père et qu'il me l'a laissée. La bibliothèque a appartenu à ma tante. Tout cela représente un passé concret qui se définit par l'héritage. C'est à moi parce que cela a appartenu à quelqu'un d'autre, ce qui me donne la notion du passé et aussi celle de la mort, car je peux me dire que quand je mourrai, je vais laisser cela à mes descendants. Dans le cas d'Haïti, où l'espérance de vie est si brève, tout est au présent, car les gens n'ont pas le temps d'accumuler des biens, sauf dans les histoires de mafia, où l'on gagne une richesse très vite, qu'on peut perdre aussi très vite, ou de politique où, du jour au lendemain, on peut se faire renvoyer comme un malpropre. Donc, la notion de profondeur de champ n'existe pas dans la mentalité d'un peuple où les gens mangent au jour le jour. Ils peuvent bien l'employer, mais ils ne savent pas ce que c'est. Cela reste un passé récent parce qu'ils ne 
peuvent pas remonter trop loin dans le temps. C'est également vrai pour l'avenir, qui est un avenir récent. Ils ne peuvent pas trop s'éloigner puisqu'ils savent très bien que la frontière des cinquante ans ne sera pas franchie par tout le monde. Tout se construit autour de cette idée. J'écris en me disant que, si je suis sincère, que je me laisse aller sans trop vouloir en faire, que je réfléchis à l'intérieur même de cet imaginaire, de cette sensibilité, je finirai par faire venir des choses qui ressemblent à ce que les gens perçoivent. C'est cela que j'aime. Je trouve cela naturel de vivre dans le présent. Ma mémoire est une mémoire présente qui ne sert qu'à me consoler. Elle a une fonction très directe liée à mon affectivité, elle ne m'apporte pas de biens.

VoIX ET IMAGES Les livres pour enfants font partie intégrante de votre univers romanesque. Était-ce important pour vous d'introduire cet univers chez les jeunes?

DANY LAFERRIÉre J'écris des livres. Les lecteurs ont tous les âges. Vous avez lu Je suis fou de Vava, vous avez bien vu que la poésie de ce livre n'est pas différente de celle des autres romans. Les textes qui sont là auraient pu se trouver dans L'énigme du retour, dans L'odeur du café ou dans un autre de mes livres. J'ai écrit trois livres - le troisième n'est pas encore terminé - qui touchent ce groupe d'âge et qui permettent aux enfants de lire sur ces thèmes: l'amour, avec Je suis fou de Vava, la mort, avec La fête des morts, et la politique, avec le prochain livre. Les enfants ont le temps de réfléchir à ces questions. Ils sont obsédés, passionnés par des questions de justice, d'injustice, de souffrance et aussi des questions relatives à l'origine, qui les ramènent à leur maman. Avec les enfants, on est en plein cœur de la politique. Ce sont les individus les plus politisés. Ils refusent toute forme d'explication face à l'injustice. Si un ami pleure, l'enfant l'amène à sa maman et lui dit: «Guéris-le. » On voit bien dans ce «guéris-le» qu'il prend le parti de l'enfant qui pleure contre celui de la mère. Cette dernière n'est qu'une employée qui est là pour guérir les gens. Il connaît la route qui l'amène à la mère, mais il ne lui appartient pas. C'est: «Guéris-le. » En même temps, les enfants peuvent être d'une violence inouïe quand ils croient que quelque chose leur appartient: «C'est ma mère.» Pour moi, ils sont au cœur de la politique. Ils font souvent preuve d'une grande violence pour certains clichés, tandis que, pour d'autres, ils sont complètement aveugles. Ils ne voient pas, par exemple, les questions de couleur, de race, et tout cela. Mais je ne leur attribue aucun angélisme, en affirmant, par exemple: «Ils sont bons.» Je soutiens plutôt que même s'ils n'ont pas les mêmes débats politiques que les adultes, ils ont la même violence. Les débats sont différents, les luttes sont différentes, mais on retrouve la même injustice, la même violence. Seulement, sur certains points, ils sont plus philosophes. C'est normal, ils ont du temps, donc ils peuvent réfléchir. La question de la mort, qui les touche puisqu'ils sont souvent confrontés, à quatre, cinq ou six ans, à la mort de leurs grands-parents, ils doivent la résoudre seuls, car les parents leur cachent la mort. Ils sont très tôt obligés de résoudre des problèmes extrêmement complexes, mais ils le font avec élégance. Il faut prendre soin des enfants, s'y intéresser. C'est un groupe de gens simplement intéressant. Pas mieux ni plus mauvais qu'un autre, mais différent.

VoIX ET IMAGES Vous avez annoncé, notamment avec la parution de Je suis fatigué en 2000, que vous aviez décidé de prendre votre retraite de l'écriture. Pourtant vous avez, depuis cette déclaration, récrit, en les modifiant considérablement, des textes publiés antérieurement, comme Cette grenade..., Le goût des jeunes filles et La chair 
du maître (dont le titre a été changé pour Vers le sud). Vous avez également écrit deux autres romans. Cette fatigue que vous ressentiez était-elle plutôt liée à votre projet d'écriture? Car on dirait que vous êtes passé à autre chose dans les deux derniers romans, comme si la fatigue était devenue le moteur d'un nouveau projet.

DANY LAFERRIĖRE Il faut qu'un monde meure pour qu'en naisse un nouveau. Et il faut l'annoncer. J'avais remarqué - ce n'est pas un commentaire après coup - que, lorsqu'on n'annonce pas la fin d'un monde, on a tendance à le faire durer, et c'est là qu'on commence à mal écrire, à se répéter. Il faut l'annoncer, cette mort. C'est le rituel des humains. Il faut dire qu'il y a funérailles en la demeure. Donc, dès que j'ai senti que ce monde était mort, j'ai dit, de façon sincère: «J'arrête d'écrire.» Ce n'était pas parce qu'il y avait chez moi une panne d'écriture; je pouvais encore écrire. Mais je n'avais pas envie de me répéter, de jouer à écrire. Et si quelqu'un veut voir cela, il n’a qu'à prendre mes livres. Ils sont liés entre eux, on a l'impression que des thèmes se répètent, que des paragraphes, des parties, des pages se répètent, mais jamais dans la même forme. La chair du maître est totalement différent de L'odeur du café, qui est totalement différent de Cette grenade..., qui est totalement différent d'Éroshima, et ainsi de suite. Ce sont des livres différents. D'un livre à l'autre, mon écriture est d'ailleurs toujours un peu plus serrée.

VoIX ET IMAGES Vous avez souvent dit que vous refusez les étiquettes que la critique vous attribue, comme celles d' «écrivain migrant», «caribéen », « haïtien » ou " québécois ». Vous avez même affirmé que vous préféreriez qu'on parle de vous comme d'un «mauvais écrivain» plutôt que comme d'un «bon écrivain migrant». Pourtant, on continue de vous accoler des étiquettes. Pour vous, celles-ci peuvent-elles faire une différence, par exemple, sur le lecteur?

DANY LAFERRIÈRE J'en ai une quand même : "écrivain japonais »! En fait, il faut dire que, comme dans beaucoup de pays, les gens dans la rue sont plus avant-gardistes que les élites. Pour une raison simple, ce sont les élites qui établissent les ordres auxquels on doit obéir, parce que les étiquettes, les conventions, sont souvent plus profitables. Mais, dans la réalité, ces considérations ne touchent pas le lecteur. Celui-ci va en librairie, il prend un livre, il en lit la quatrième de couverture, il le feuillette, il en regarde un autre et il choisit finalement le livre qu'il aimerait lire. Pour lui, ces étiquettes, même quand on les met dans les librairies, en aménageant des sections selon le genre attribué aux livres, n'ont pas d'importance. Le lecteur québécois ne s'intéresse pas à ces étiquettes. Il reste dans la grande tradition de la bibliothèque et de la librairie qui sont, précisément, des endroits où on peut rencontrer des gens qu'on ne connaît pas, où on peut lire des gens qu'on ne connaît pas. Les étiquettes sont liées aux institutions. C'est sans doute un peu plus fort au Québec qu'ailleurs, parce que ce débat est ici plus profond, en ce qu'il est lié à la notion d'indépendance. Le Québec a été poussé à la marge à tel point qu'il a dû définir son identité par la distinction, en se percevant comme une société distincte. C'est normal, alors, qu'il distingue, qu'il continue à distinguer et qu'il ne comprenne pas que l'autre ne veut pas de distinction, parce que le Québec a gagné de haute lutte d'être une société distincte. Alors quand tu lui dis, toi, que tu ne veux pas être distinct, il se demande ce que tu veux. C'est comme quand on dit: "Les immigrants veulent que les gens les appellent des Québécois. Les Québécois veulent que les immigrants soient intégrés.» Et pourquoi 
ils ne les appellent pas des Québécois? Ils continuent à les distinguer, parce que tout le cœur de l'analyse sociétale est lié à cela. Alors, quand tu leur dis : «Je ne veux pas être vu comme un immigrant», ils demandent: "Mais comment on doit t'appeler? -Qu'on m'appelle un Québécois. - Pourquoi tu veux t'intégrer, quand nous, on veut se distinguer? » C'est là, je trouve, toute la dichotomie, toute l'opposition qui fait que le Québec a continué à multiplier les distinctions: Noir, Caribéen, migrant... On n'arrive pas à se défaire de cela. D'ailleurs, les Québécois veulent de plus en plus l'intégration, mais tout en gardant la distinction, parce que, pour eux, intégrer avec distinction veut dire une richesse. C'est-à-dire qu'ils refont le fédéralisme: il y a un centre, la société de souche, et on fait une fédération avec des satellites autour.

VoIX ET IMAGES Si on devait vous mettre une autre étiquette que celle d'écrivain japonais, ce serait celle de moraliste. Vous avez vraiment ce génie de la formule bien ramassée, bien ciselée, qui est la marque des moralistes français et de leurs successeurs. Ceci semble particulièrement vrai dans L'énigme du retour. Seriez-vous à l'aise avec une étiquette comme celle-là?

DANY LAFERRIĖRE Une seule étiquette, ce n'est pas bon, mais beaucoup d'étiquettes, il n'y a pas de problème. Oui, bien sûr, du moment que je ne suis pas moralisateur. Je rêverais aussi d'une proximité avec Diderot. Et c'est là la faiblesse et la force, si je peux commenter mon propre travail, de L'énigme du retour, parce que, moi qui ne veux pas être vu, qui ai cherché dans la plupart de mes livres, contrairement à ce que j'ai fait dans mes chroniques, à ne pas faire de phrases qui frappent, je me suis laissé aller dans celui-là. Mais j'ai pu le faire parce que j'ai pris une autre forme, celle de la poésie, qui a fait que cela est passé inaperçu. D'où le succès et, peut-être, l'échec de ce livre... on ne sait jamais. Le temps nous dira si ces maximes étaient abusives ou bien si elles fonctionnent bien, parce que ces choses-là sont très délicates. Ce sont des choses qui retiennent l'attention sur le moment, par le côté nouveau de l'affaire, et, au fil des années, ça s'effrite et ça finit par dater.

voix ET IMAGES Ce livre vous a pourtant valu le prix Médicis, ce qui est un bon départ pour la pérennité du roman. Comment recevez-vous ce prix? Est-ce qu'il change quelque chose pour vous, à ce moment de votre carrière?

DANY LAFERRIĖRE Non, je pense que le prix concerne avant toute chose le lecteur. Un prix a une signification, dans le sens profond du terme, au début d'une carrière, si l'on peut dire, là où vraiment cela peut endommager ou donner un élan. Pour ma part, je pense que ce prix n'a d'impact véritable que sur le lectorat, la critique, la presse, les institutions, soit pour les faire venir en plus grand nombre, soit pour leur faire regarder la chose différemment. C'est certain que c'est la meilleure situation, d'avoir un prix. En même temps, j'ai écrit dix-neuf livres qui ne sont pas si disparates que ça, puisqu'on a pu les rassembler sous le titre d'« Autobiographie américaine». Le reste, ce sont des réflexions autour de cette œuvre. Même mes livres pour enfants sont des satellites de L'odeur du café et des autres. Donc, tout cela semble assez cohérent. Il y a là un univers. C'est l'univers qui va dévorer le prix. Le prix n'a d'impact sur un écrivain que lorsqu'il peut dévorer l'écrivain, c'est-à-dire quand il peut faire en sorte que l'écrivain se dise: «Il faut que j'écrive un livre qui montre que je suis digne d'avoir reçu ce prix.» Ou, au contraire, quand il ne peut plus écrire parce qu'il a peur de ne pas être à la hauteur de ce prix et de passer, aux yeux des autres, pour un imposteur. 
Moi, j'ai déjà ma démarche, c'est trop tard. Je ne le dis pas dans le sens que cela devait arriver plus tôt, je dis c'est trop tard, le mal a déjà été fait. Mais cela m'a touché, car je ne voulais même pas le publier, ce livre, donc c'est arrivé comme un cadeau.

VoIX ET IMAGES Vous ne teniez vraiment pas à publier ce livre?

DANY LAFERRIẼRE Non, je l'ai envoyé chez Boréal et chez Grasset en leur disant: «Si cela vous intéresse...» En fait, pour moi, c'était un de ces trucs qui doivent se retrouver dans des tiroirs, parce que cela sert à écrire, à percevoir où j'en suis avec certaines émotions. Les éditeurs m'ont dit: «Non, ce serait intéressant, ça permettrait aussi aux gens de voir tout l'éventail des émotions et des thèmes qui traversent vos autres livres.»

VoIX ET IMAGES Il doit y avoir moins de retenue, d'autocensure, quand on écrit un livre qu'on destine au tiroir?

DANY LAFERRIĖRE Ha, mais oui! Bien sûr, ce n'est pas la première version qui est publiée. J'ai fait quelques concessions - pas aux éditeurs, mais à moi - en rendant la chose plus fluide, en rendant aussi les textes plus narratifs, afin de ne pas trop agresser le lecteur. Je voulais que les gens qui n'aiment pas lire lisent cet ouvrage. Mais je savais que si je gardais uniquement la forme poétique, ils ne l'auraient pas lu, parce que le livre aurait été un objet non identifié. Donc, j'ai rédigé des textes plus narratifs, ce qui les rendait plus accessibles. Quand on écrit pour ne pas être lu, on écrit de façon plus intime, et les informations sont plus elliptiques. Il y a certaines fois où j'ai semé des indices qui permettent de comprendre, de passer d'un monde à un autre. Sans en faire trop, mais sans sortir, non plus, du projet littéraire.

voix ET IMAGES Même si vous affirmez que vous avez mélangé les genres à l'intention du lecteur, ce procédé n'est pas nouveau dans votre œuvre. Par exemple, il y a des haïkus dans Éroshima, des recours au scénario de film dans Le goût des jeunes filles... DANY LAFERRIÉre Et aussi dans Cette grenade..., ainsi que dans Chronique de la dérive douce. On y retrouve toutes sortes de mélanges. Ce qui est intéressant, avec L'énigme du retour, c'est le phénomène de la réception, à cause du succès de lecture qui est nouveau pour moi. J'ai déjà eu beaucoup de succès public, mais les gens ne lisaient pas vraiment mes livres. Et là, les gens découvrent, et comme ils découvrent assez tard, ils sont obligés de se faire des opinions assez péremptoires. Ils disent que c'est nouveau. Même les critiques l'ont fait, surtout ceux qui écrivent dans les journaux. Alors que la plupart de mes livres ont tous été singuliers dans leur manière. J'ai souvent changé de thème, de ville, d'émotion, d'âge et d'époque. Ce qui rassemble le tout, c'est peut-être un regard, une façon de regarder les choses. Mais ils ont cru que ce regard, dans L'énigme du retour, était différent de celui présent dans L'odeur du café ou dans Comment faire l'amour... Ce qui est faux, puisqu'en regardant l'ensemble, on voit bien comment c'est modelé. Lorsqu'on me dit: "Vous n'avez jamais écrit comme ça avant» ou bien qu'il s'agit d'un «livre de maturité», je sursaute. Ou bien, lorsqu'on dit: «C'est un livre grave comme vous n'en avez jamais écrit. » Mais ce n'est pas grave du tout à côté du Cri des oiseaux fous. Quand on dit que la forme de ce livre est nouvelle, on semble perdre de vue que Chronique de la dérive douce était encore plus risqué, parce qu'il n'y avait même pas ces formes et ces segments narratifs; c'était plus direct. Ces critiques m'apparaissent donc un peu superficielles.

VoIX ET IMAGES Espérons que ce dossier permettra de faire connaître votre œuvre plus en profondeur. 